\title{
SIMULATOR ASSISTED ENGINEERING - APPLICATIONS IN NUCLEAR ENGINEERING EDUCATION AT KHALIFA UNIVERSITY
}

\author{
Ahmed K. Alkaabi ${ }^{1}$, Mohamed Ali ${ }^{1}$, Ho Joon Yoon ${ }^{1}$, Oussama Ashy ${ }^{2}$ \\ ${ }^{1}$ Department of Nuclear Engineering, Khalifa University of Science and Technology, \\ P.O. Box 127788, Abu Dhabi, United Arab Emirates \\ ${ }^{2}$ Western Services Corporation \\ 7196 Crestwood Blvd \#300, Frederick, MD 21703, United States \\ ahmed.alkaabi@ku.ac.ae,mohamed.ali1@ku.ac.ae, hojoon.yoon@ku.ac.ae, \\ ashyo@ws-corp.com
}

\begin{abstract}
The Generic Pressurized Water Reactor (GPWR) simulator has been used in the Nuclear I\&C Laboratory at Khalifa University (KU) since 2013 to improve student performance in nuclear engineering that is a multidisciplinary field involving nuclear reactor physics, thermodynamics, fluid mechanics, thermal hydraulics, radiation, etc. The simulator, developed by Western Service Corporation, has been integrated as a teaching and educational tool in different Engineering Programs at KU (Mechanical and Nuclear engineering). This lab is used in an undergraduate course where students apply the knowledge taught from different courses such as nuclear systems, fuel cycle, thermal hydraulics, safety principle, and control functions through a virtual operating NPP simulator. This real-time, full scope and high fidelity simulator allows to perform different operating conditions such as plant startups, shutdowns, and load maneuvers; as well as normal and abnormal plant transients, and critical scenarios and accidents. Since its installation in the Nuclear I\&C Laboratory at KU in 2013, thirty students have benefited from this learning simulator. The main skills and learning outcomes expected to be achieved by students through the using of this tool are (i) ability to describe different NPP components and understand different process occurring in different subsystems, (ii) explain and apply safety principles and protective protocols, and (iii) analyze and interpret the plant behavior during transient operations and when severe accidents happen.
\end{abstract}

KEYWORDS: Simulated Assisted Engineering, Nuclear Engineering Education, Pressurized Water Reactor

\section{INTRODUCTION}

Nuclear Engineering (NEng) is a multidisciplinary field involving nuclear reactor physics, thermodynamics, fluid mechanics, radiation, etc. The complexity and diversity of problems and concepts related to NEng in general and Nuclear Power Plant (NPP) operation in particular make nuclear engineering education a real academic challenge. The difficulty of problems representation in combination with complex mathematical and conceptual analysis encountered in NEng makes it extremely difficult for students to learn. To date, numerous educational tools and methods have been developed to improve student performance in nuclear engineering. One of these advanced learning methods is Simulator Assisted Engineering (SAE). 
For this purpose, a learning simulator, Generic Pressurized Water Reactor (GPWR), was installed in the Nuclear I\&C Laboratory at Khalifa University (KU) in 2013, with additional upgrading in 2015 and 2019. The simulator, developed by Western Service Corporation, has been integrated as a teaching and educational tool in different Engineering Programs at KU (Mechanical and Nuclear engineering). It is used in an undergraduate course where students apply the knowledge taught from different courses such as nuclear systems, fuel cycle, thermal hydraulics, safety principle, and control functions through a virtual operating NPP simulator. It is also used by MSc students to conduct their research projects. This real-time, full scope and high fidelity simulator allows to perform different operating conditions such as plant startups, shutdowns, and load maneuvers; as well as normal and abnormal plant transients, and critical scenarios and accidents. Since its installation in the Nuclear I\&C Laboratory at KU in 2013, thirty students have benefited from this learning simulator. The main skills and learning outcomes expected to be achieved by students through the using of this tool are (i) ability to describe different NPP components and understand different process occurring in different subsystems, (ii) explain and apply safety principles and protective protocols, and (iii) analyze and interpret the plant behavior during transient operations and when severe accidents happen. Class Hands-on assignments using simulator includes:

- Manual Reactor Trip

- Simultaneous Trip of Main or all Feedwater Pumps

- Simultaneous Closure of all Main Steam Isolation Valves

- Simultaneous Trip of all Reactor Coolant Pumps

- Trip Single Reactor Coolant Pump

- Main turbine trip without reactor SCRAM, at 30\%

- Maximum rate power ramp from $100 \%$ down to $75 \%$ and back up to $100 \%$

- Maximum size reactor coolant system rupture combined with loss of all offsite power

- Maximum size unisolable main steam line rupture

In the present paper, we present some examples of the studied scenarios and different relevant outputs that student can handle and analyze.

\section{Simulated scenarios using the simulator}

\subsection{Manual reactor trip}

The simulator GPWR allows to study the case of a manual reactor trip. Using a defined scenario, students can monitor different operation parameters during the test and verify all components status. They can verify mainly the position of the control rod which should be at the bottom of the reactor core, the turbine and steam generator valves, and the generator output. At the end of the process, a file is written and saved containing data about different parameter behaviors. The students can later analyze this output to understand the effect of the activation of the manual trip on the reactor behavior. Some of the parameters that can be analyzed are:

i- $\quad$ the neutron flux: it should respond with an immediate decrease in flux from $100 \%$ to delayed neutron flux level in such reactor. This behavior can be verified by the students using the temporal evolution of the neutron flux as illustrated by Figure 1. 


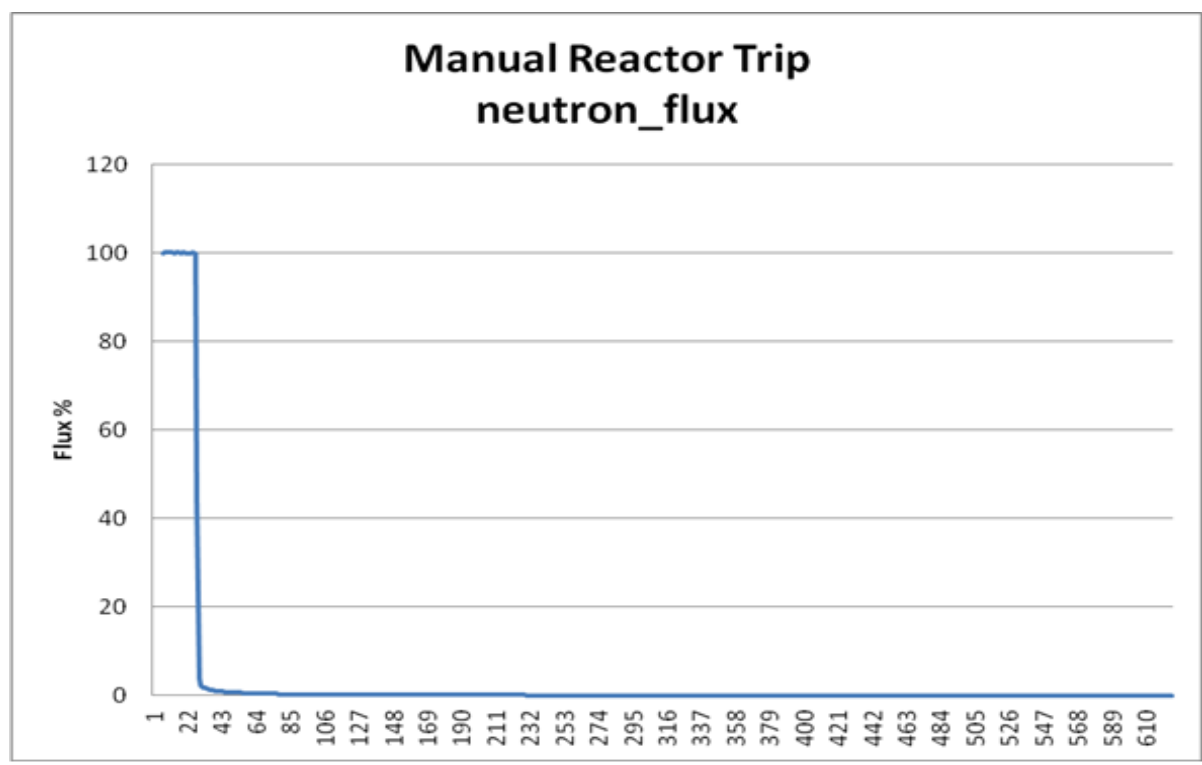

Figure 1: Evolution of the neutron flux during the manual reactor trip test

ii- $\quad$ average temperature: during this transient test, it should decrease following the neutron flux behavior. In case of unexpected evolution, steam dump will be automatically activated to maintain the average temperature at its no-load value.

iii- pressurizer level: as the reactor is cool-down, the specific volume of the circulating coolant decreases and causes a pressure decrease at the pressurizer. To maintain this pressure at its normal operating level, the charging flow control valve is automatically open. Students can verify this behavior analyzing data as shown by Figure 2 .

iv- $\quad$ steam generator pressure: it is an important parameter to control. It should rapidly rise following the main turbine behavior. In order to keep it at no-load conditions, the steam dumps firstly open causing an immediate decrease in steam pressure and then they gradually close to bring the pressure at the desired level. 


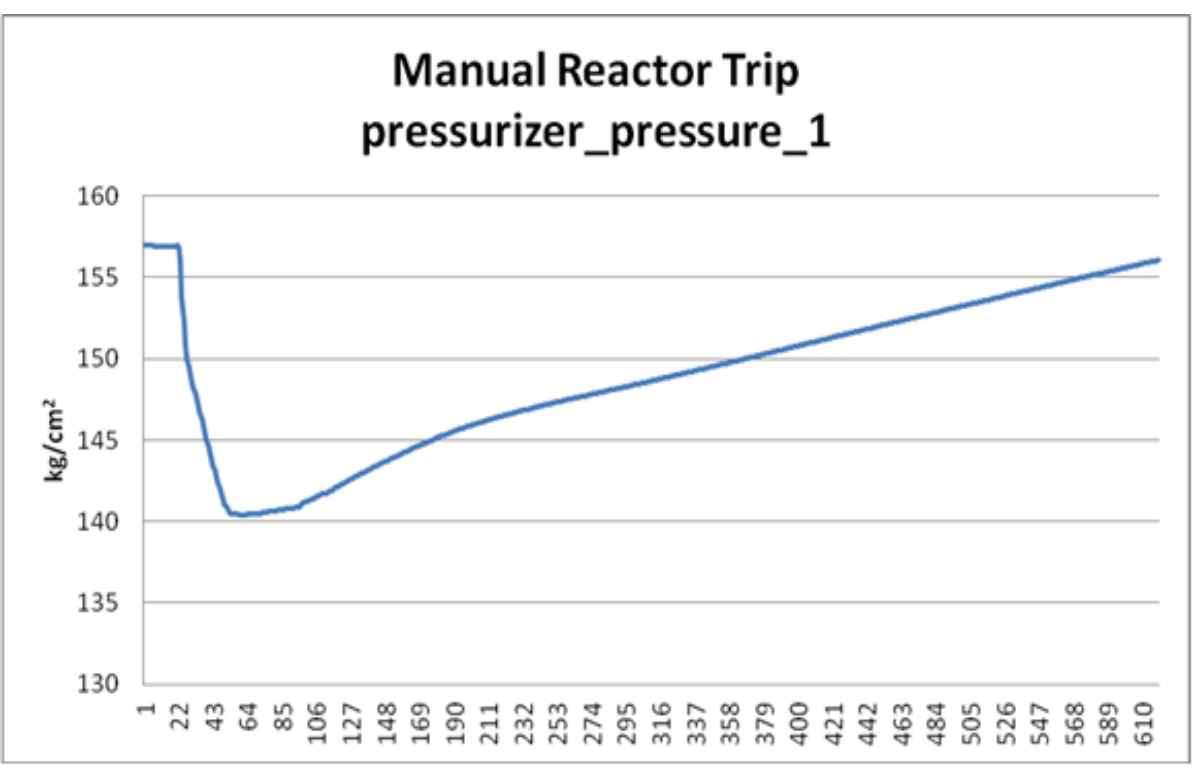

Figure 2: Temporal evolution of the pressurizer pressure during the manual reactor trip test.

\subsection{Simulation of a simultaneous trip of main or all feedwater pumps}

During this test, the reactor operates at $100 \%$ power for a short period and then the malfunctions are inserted. The simulator runs for 10 minutes and different parameters can be monitored and saved to be analyzed. Students can verify different operation parameters such as the generator power and the pressure in the secondary side of the steam generator, turbine and auxiliary motor driven feed pumps.

i- $\quad$ Feedwater flow: as shown by Figure 3, the total feedwater flow for all steam generators decreases immediately to zero and remains until the end of the test.

ii- $\quad$ Steam generator pressure: the behavior of the pressure during this event can be divided into three regions. First, it increases due to the loss of the feedwater and the deviation of the steam from the feedwater pumps to the main turbine. Second, the pressure decreases after the open of steam dumps. Finally, the steam dumps gradually close and the steam generator pressure is brought to no-load conditions. The temporal evolution of the steam generator pressure is illustrated by Figure 4. 


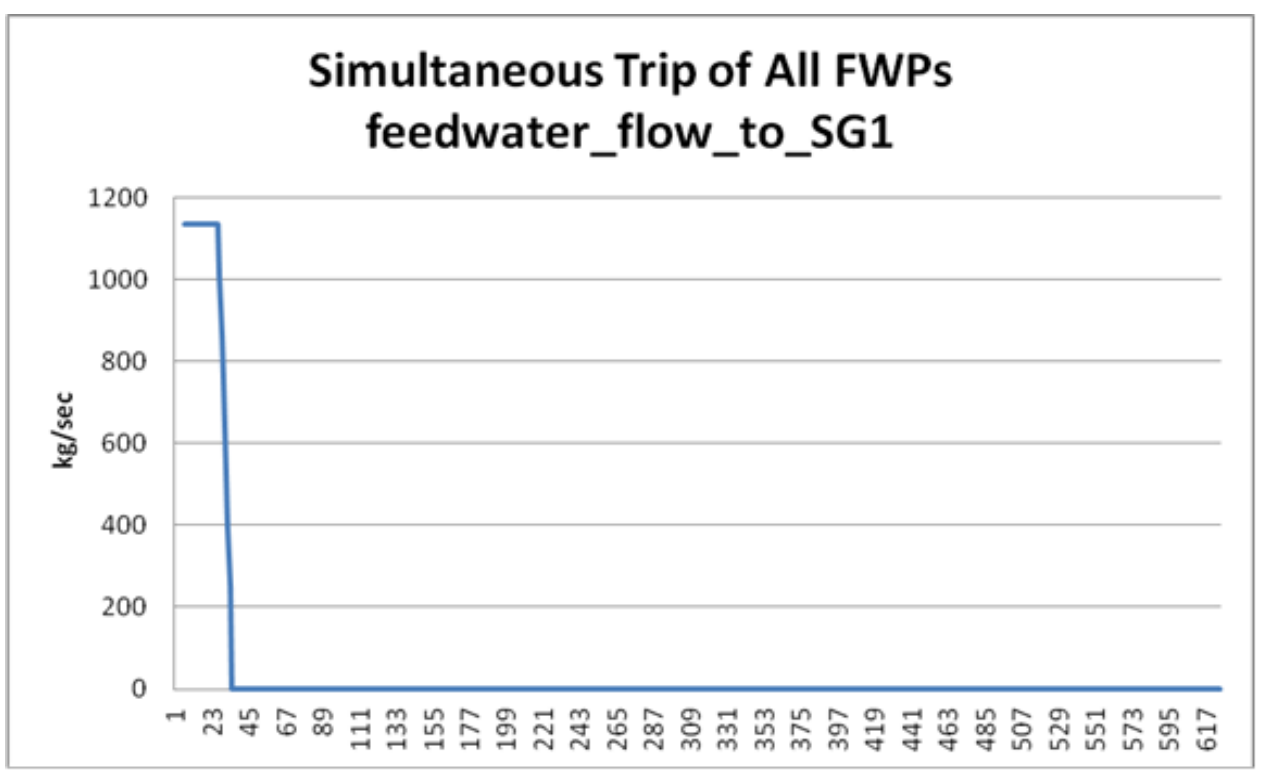

Figure 3: Feedwater flow during simultaneous trip of all feedwater pumps event

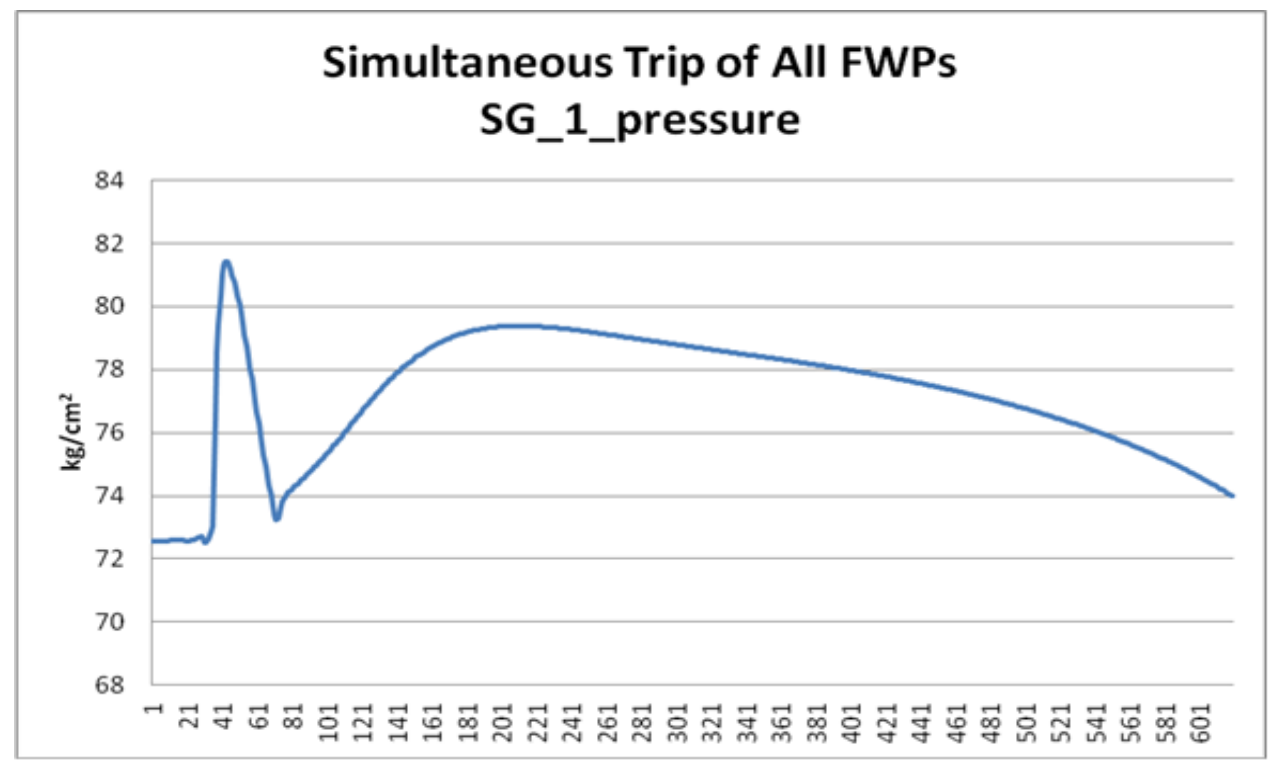

Figure 4: Response of the steam generator pressure during the simultaneous trip of all feedwater pumps event

\subsection{Simulation of a simultaneous trip of all reactor coolant pumps}

In this event, students are asked to monitor and verify the reactor behavior when all reactor coolant pumps are stopped. The duration of the test is 10 minutes during which data are recorded. During the test, the turbine main stop valves close and turbine trip is activated by reactor protection system. The generator power decreases immediately to a negative value and after generator breakers trip open it remains at $0 \mathrm{MW}$. The steam dump controller demand increases to $100 \%$ and the steam dump valves will be operated to maintain average temperature at the no-load value.

Later, analysis of different parameters is requested such as: 
i- $\quad$ The neutron flux: due to trip of the coolant pumps, moderator temperature and fuel temperature will begin to increase providing net negative reactivity. Therefore, the reactor trip occurs and the neutron flux decreases from $100 \%$ to delayed neutron flux level in a subcritical reactor.

ii- $\quad$ Pressurizer pressure: it decreases immediately after coolant pump closing due to the increase in density of the reactor coolant. Then, it starts to increase after establishment of natural circulation in addition to the activation of the pressurized backup heaters. It continues increasing until reaching the set-point of the pressurizer power-operated relief valves, as shown in Figure 5.

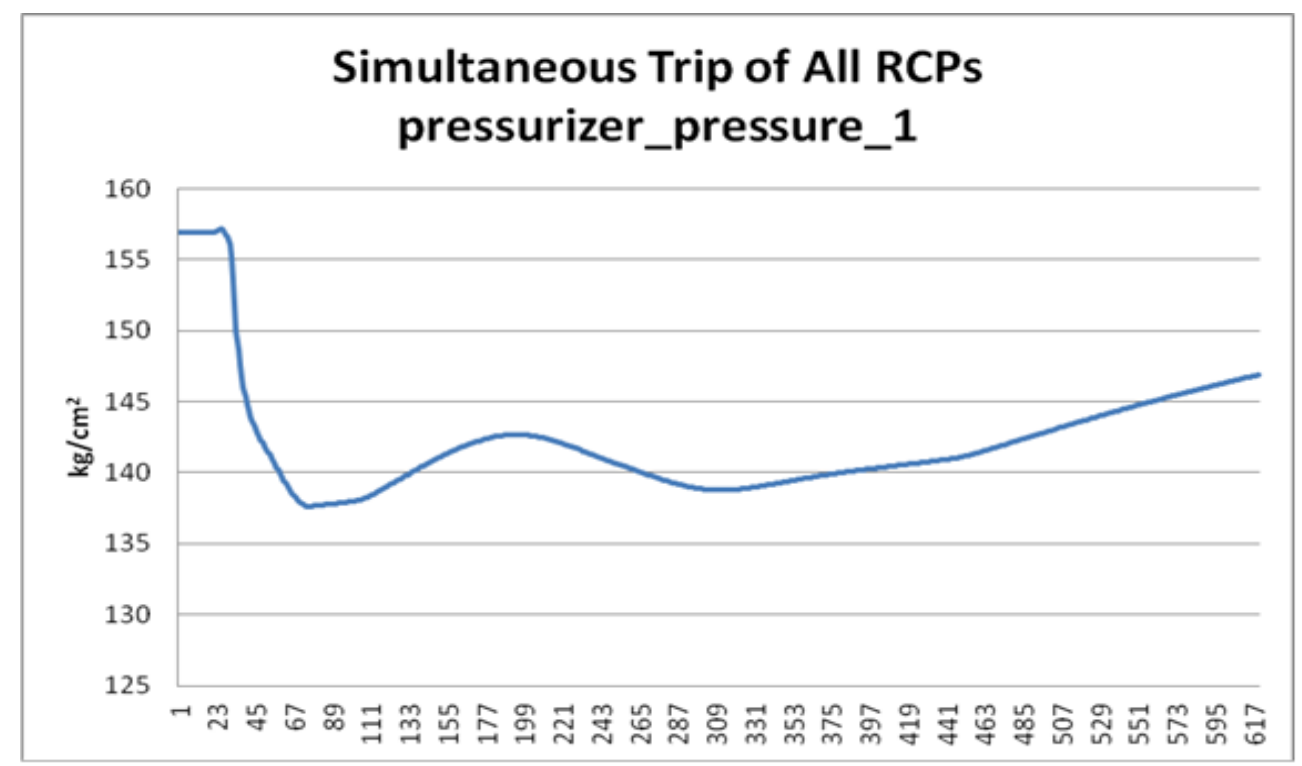

Figure 5: Evolution of the pressurizer pressure during a simultaneous trip of all reactor coolant pumps scenario.

\subsection{Simulation of the maximum size reactor coolant system rupture combined with loss of all offsite power}

During this scenario, students can follow the different component behavior due to a rupture of coolant system. They can verify that all control rods drop to the bottom of the reactor core, the turbine main stop valves close, and pressurizer level decreases to zero as answer to these malfunctions. As primary parameters of interest, students can analyze:

i- $\quad$ The containment temperature and pressure: they increase rapidly when the event starts and then decrease due to the effects of quench spray and recirculation spray cooler operation. The temporal evolution of the temperature is shown in Figure 6.

ii- $\quad$ Steam generator level: its response will globally follow a reactor trip. As shown in Figure 7, it is characterized by the fluctuation during some period of the test. It will stabilize due to the decrease of the decay heat and be maintained by manually throttling auxiliary feedwater. 


\section{CONCLUSIONS}

According to the encouraging feedback of students and instructors involved on the use of the GPWR simulator, this learning method is found to be more efficient and attractive: students feel more comfortable with the direct application of the fundamental knowledge on real engineering problems. Therefore, and in order to enhance the efficiency of this educational tool, KU keeps updating and upgrading the simulator by integrating advanced tools allowing better use for education, training and R\&D. For example, RELAP-3D engineering grade thermal-hydraulic model, developed by Idaho National Laboratory for real light water reactor analysis, is recently integrated and used to simulate real-time thermal hydraulic behavior of different components.

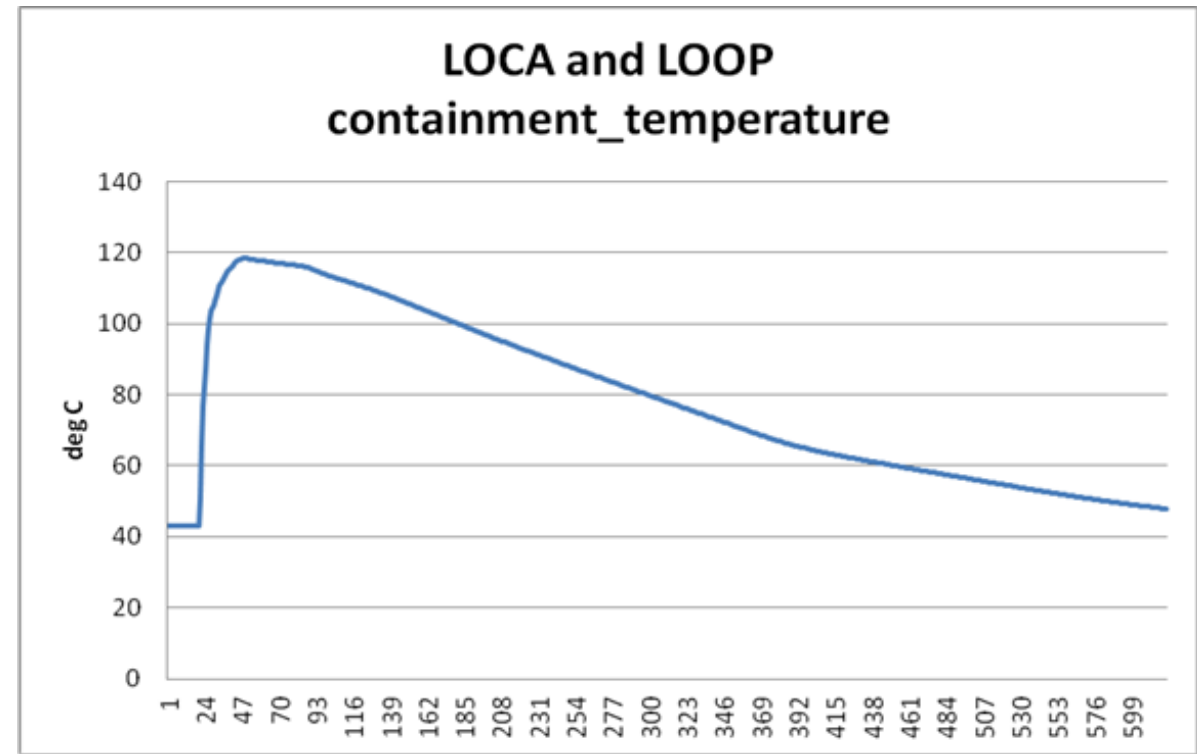

Figure 6: Temperature containment during the maximum size reactor coolant system rupture event.

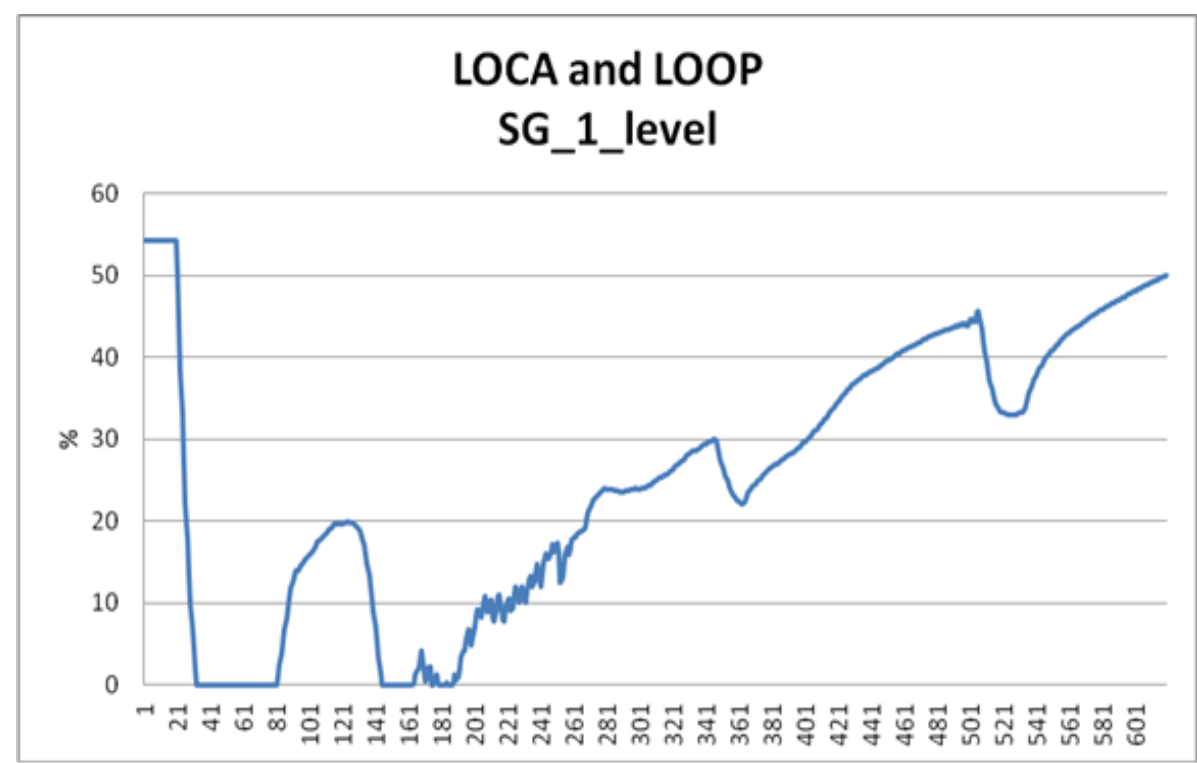

Figure 7: Oscillating behavior of the steam generator level during the maximum size reactor coolant system rupture event. 